\title{
Education and training policies for research integrity: Insights from a focus group study
}

\author{
Krishma Labib ${ }^{1, *}$, Natalie Evans ${ }^{1}$, Rea Roje ${ }^{2}$, Panagiotis Kavouras ${ }^{3}$, Andrea Reyes Elizondo ${ }^{4}$, \\ Wolfgang Kaltenbrunner ${ }^{4}$, Ivan Buljan ${ }^{2}$, Tine Ravn $^{5}$, Guy Widdershoven ${ }^{1}$, Lex Bouter ${ }^{6,7}$, \\ Costas Charitidis ${ }^{3}$, Mads P. Sørensen ${ }^{5}$ and Joeri Tijdink ${ }^{1,7}$
}

\begin{abstract}
${ }^{1}$ Department of Ethics, Law and Humanities, Amsterdam Public Health Institute, Amsterdam UMC, Vrije Universiteit Amsterdam, Amsterdam, The Netherlands, ${ }^{2}$ Department of Research in Biomedicine and Health, School of Medicine, University of Split, Split, Croatia, ${ }^{3}$ Department of Materials Science and Engineering, School of Chemical Engineering, National Technical University of Athens, Athens, Greece, ${ }^{4}$ Centre for Science and Technology Studies, Leiden University, Leiden, The Netherlands, ${ }^{5}$ Department of Political Science, Aarhus University, The Danish Centre for Studies in Research and Research Policy, Aarhus, Denmark, ${ }^{6}$ Department of Epidemiology and Data Science, Amsterdam Public Health Institute, Amsterdam UMC, Vrije Universiteit Amsterdam, Amsterdam, The Netherlands and ${ }^{7}$ Department of Philosophy, Vrije Universiteit Amsterdam, Amsterdam, The Netherlands
\end{abstract}

*Corresponding author. E-mail: k.labib@amsterdamumc.nl

\begin{abstract}
Education is important for fostering research integrity (RI). Although RI training is increasingly provided, there is little knowledge on how research stakeholders view institutional RI education and training policies. Following a constructivist approach, we present insights about research stakeholders' views and experiences regarding how research institutions can develop and implement RI education and training policies. We conducted thirty focus groups, engaging 147 participants in eight European countries. Using a mixed deductive-inductive thematic analysis, we identified five themes: (1) RI education should be available to all; (2) education and training approaches and goals should be tailored; (3) motivating trainees is essential; (4) both formal and informal educational formats are necessary; and (5) institutions should take into account various individual, institutional, and system-of-science factors when implementing RI education. Our findings suggest that institutions should make RI education attractive for all and tailor training to disciplinary-specific contexts.
\end{abstract}

Key words: research integrity; responsible conduct of research; education; training; research institutions; institutional policies; research stakeholders

\section{Introduction}

There is a growing awareness of the importance of research integrity (RI) for producing high-quality and relevant research (Nature, 2019). RI can be defined as doing research according to high ethical, professional, and methodological standards (Boehme et al. 2016). RI education is considered to be crucial in fostering RI and in reducing misbehaviors-both serious (e.g. fabrication, falsification, and plagiarism) and minor (e.g. questionable research practices such as hypothesizing after the results are known; Hermerén et al. 2019). It is widely acknowledged that research institutions are responsible for providing good-quality RI education and training (Resnik and Shamoo 2011; Forsberg et al. 2018; Fanelli 2019). Although there are various ways to differentiate between 'education' and 'training' (Masadeh 2012), in this paper, we use the term 'RI education' to refer broadly to all approaches-both formal and informal-used to develop knowledge, skills, moral values, and understanding of RI, whereas we refer to 'RI training' when discussing formal instructional events or programs used for RI education (e.g. courses and workshops). RI training is, thus, a crucial part of RI education.
In the past decades, many stand-alone RI courses have been developed globally, mostly aimed at $\mathrm{PhD}$ students, using a diversity of training designs, approaches, and organization methods (Kalichman 2013; Boehme et al. 2016; Fanelli 2019; Abdi et al. 2021). However, it is increasingly recognized that RI education entails more than isolated training events. It requires addressing various target groups-rather than merely focusing on junior researchers-as well as a continuous effort by both institutions and individual researchers (Kalichman 2007; Evans et al. 2018; Fanelli 2019). Therefore, a more systematic institutional approach to RI education is needed to ensure the relevance for stakeholders and to develop the evidence base related to different approaches and their effectiveness. In particular, it would be helpful for institutions to develop a comprehensive RI education and training policy outlining the overarching institutional RI education strategy, as well as the concrete plans and procedures needed to implement it. Indeed, RI education has been recently highlighted as one of nine important topics to address in institutional RI policies and-in Europe-is now actively promoted by the European Commission (Mejlgaard et al. 2020; SOPs4RI 2020).

(c) The Author(s) 2021. Published by Oxford University Press.

This is an Open Access article distributed under the terms of the Creative Commons Attribution License (https://creativecommons.org/licenses/by/4.0/), which permits unrestricted reuse, distribution, and reproduction in any medium, provided the original work is properly cited. 
While there are a variety of RI educational tools currently available (Pizzolato et al. 2020), much of the existing literature on RI education is quantitative and focuses on studying the evaluation of single trainings (Anderson et al. 2007; Powell et al. 2007; Godecharle et al. 2014; Marusic et al. 2016; Watts et al. 2017; Antes et al. 2018, 2009; Fanelli 2019; Mejlgaard et al. 2020). Based on the little evidence available, much of which is considered to be of poor quality (Marusic et al. 2016), it is not clear whether, and if so which, RI training approaches are valuable in changing stakeholders' behaviors related to RI (Powell et al. 2007; Antes et al. 2009; Marusic et al. 2016; Science Europe 2017; Fanelli 2019). A possible explanation for the lack of good-quality available evidence is that there is no consensus about which learning aims RI education should approach, and hence, which outcomes evaluations should measure (Fanelli 2019). Several educational approaches have been discussed and problematized as potentially suitable such as reducing misconduct, improving RI knowledge and skills, internalizing RI values, and navigating the gray areas of research (DuBois 2004; Powell et al. 2007; Geller et al. 2010; Kalichman 2014; Fanelli 2019). It is thought that RI education likely needs to incorporate both formal training and informal educational approaches (e.g. through responsible mentorship; Kalichman 2007; OECD Global Science Forum 2007; Alfredo and Hart 2011; Satalkar and Shaw 2018), although there is little insight available about how education should be organized to optimize the benefits of different educational approaches. For instance, it remains unclear whether mandatory RI educational events are desirable in ensuring participation in RI education for various target groups or whether other-and if so, which-incentives are needed (Fanelli 2019).

Although education is seen as crucial in fostering RI, the literature suggests that training by itself will likely be insufficient in influencing researchers' behaviors (Aubert Bonn and Pinxten 2021). This is because researchers' behaviors are not only influenced by their awareness and attitudes regarding RI, but also the research system in which they operate, as well as their local research culture (Titus and Bosch 2010; Joynson and Leyser 2015; Forsberg et al. 2018; Rifai et al. 2019). In fact, some have even criticized the emphasis on education and training in the field of RI, arguing that a stronger emphasis is needed on the underlying factors and different stakeholders that influence researchers' behaviors rather than focusing on individual researchers (e.g. Aubert Bonn and Pinxten 2021). There is little discussion about how institutions can successfully develop and implement institutional policies on RI education, which adequately take into account these considerations. Furthermore, there are few qualitative studies on how researchers and other stakeholders perceive and engage with RI education, even though such insights could provide needed guidance for institutions on how to develop engaging RI education and training, and incentivize participationparticularly considering the weak evidence base related to RI education. Such a constructivist approach is important for generating relevant insights with stakeholders for developing and implementing meaningful institutional policies on RI.

A set of American focus groups with medical postdoctoral researchers from 2008 suggests that researchers prefer the use of case study discussions in training to address their day-today RI dilemmas (Alexander and Williams 2008). Two recent European focus group projects showed support among diverse research stakeholders for continuous RI education across academic ranks and for everyone involved in the research process (Evans et al. 2018; Pizzolato and Dierickx 2021). Another European stakeholder consultation with RI experts resulted in the recommendation that RI education should consist of a core curriculum on RI basics (e.g. on what are questionable research practices) and a follow-up advanced curriculum to further deepen RI insights (e.g. how to prevent questionable research practices in their daily research; ENERI 2017).

While these reports provide important insights about various research stakeholders' perceptions of RI education and training, it is not clear to what extent the findings apply across different countries and disciplinary fields. Furthermore, the perspective of researchers from fields other than $\mathrm{RI}$ is particularly unexplored. Yet, following a constructivist approach, understanding stakeholders' diverse research perspectives, views, needs, and preferences about realities on the ground is crucial for developing and implementing meaningful institutional policies on RI. Therefore, in this article, we report on the results of a European focus group study with research stakeholders from various disciplines, to delve deeper into the research question: What are researchers' and other stakeholders' views and preferences regarding how research institutions can develop and implement better education and training policies?

\section{Methods}

This article is based on data from a focus group study that has been preregistered on the Open Science Framework (OSF): https://osf.io/kdtnx/ (Sørensen et al. 2021). The full data analysis protocol can be found here: https://osf.io/jntck/. The focus group study is part of the Standard Operating Procedures for Research Integrity (SOPs4RI) project (www.sops4ri.eu), which aims to develop guidance for research institutions and funders on how to foster RI. Since the insights from the focus groups were intended to inform the guidance being produced in the SOPs4RI project, we intended to obtain practical information, based on stakeholders' views and experiences, that would be valuable for institutions in developing policies on RI education. The research was approved by the Research Ethics Committee of Aarhus University (https://osf.io/kdtnx/). Information about the goals and procedure of the study were sent to participants at least a week before the focus groups took place, and informed consent was obtained before or at the start of each focus group.

\subsection{Study design}

We conducted thirty focus groups in various parts of Europe with research stakeholders from the medical, natural, and social sciences, and the humanities (Table 1 ). The focus groups aimed to provide interpretations and viewpoints of stakeholders, including perceived effects of training, based on experience, as input for guidance on RI education and training for research institutions. Each focus group started with an open discussion about RI policies, followed by a more indepth discussion of two or three RI topics, and ended with a sorting exercise. The interviews lasted 1.5-2 hours. The topic RI education and training was addressed in depth in eight focus groups, in which we asked participants to share their views on what measures institutions can take to develop RI 
Table 1. Composition of focus groups.

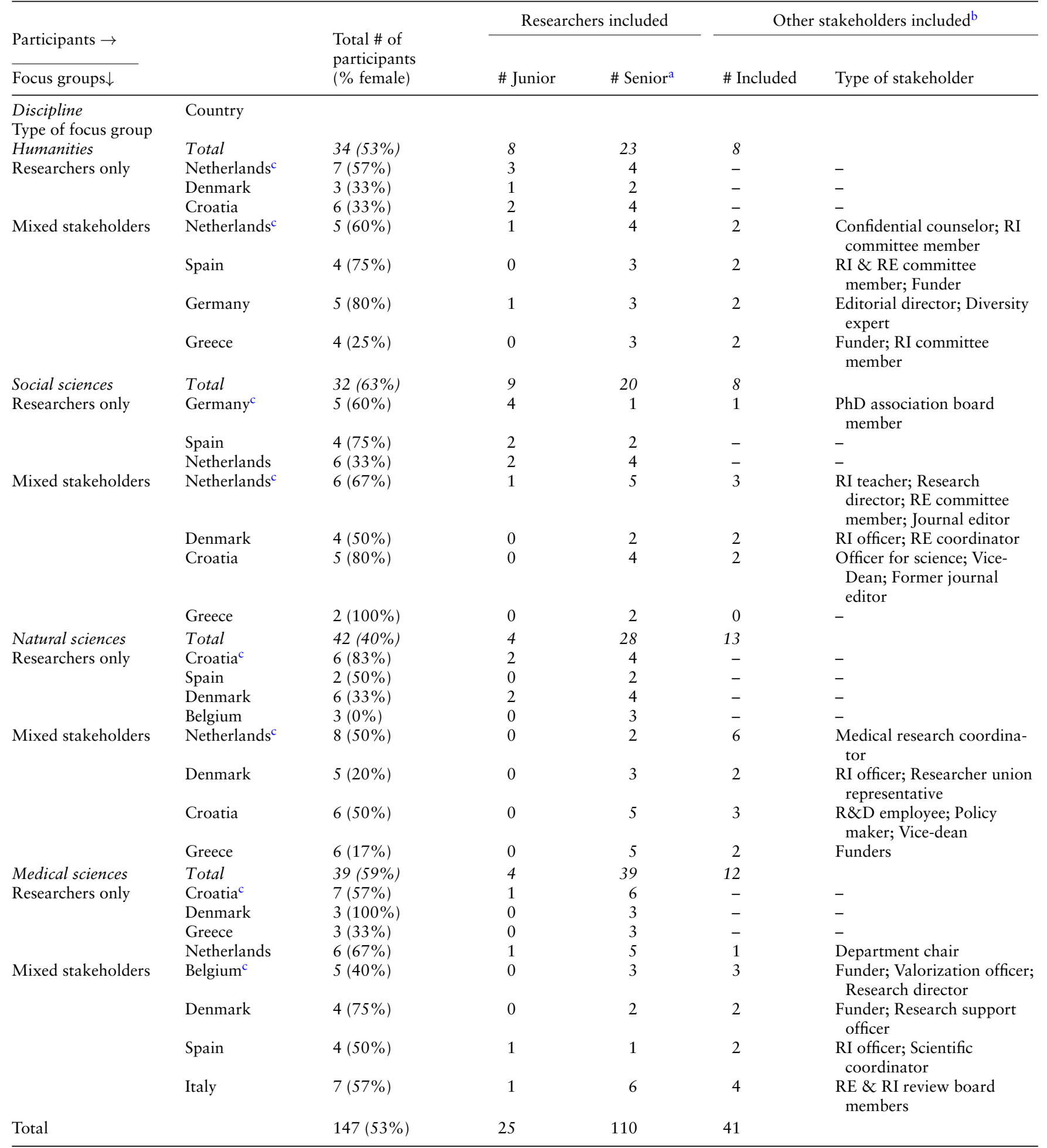

${ }^{a}$ We considered a researcher as being 'senior' if they held a tenure position (which we assumed when participants had a position as an 'assistant, associate or full professor'), or-in case we did not have this information-if participants had 5 or more years of research experience post obtaining a doctorate. We considered a researcher as being 'junior' if they did not yet hold a tenure position (which we assumed when they had a position as a 'PhD student', 'junior researcher', or 'postdoctoral researcher'), or-in case we did not have this information, if they had less than 5 years of research experience post obtaining a doctorate.

${ }^{b}$ Please note that some participants represented more than one role (i.e. represented both a researcher and other type of stakeholder, and/or represented multiple other types of stakeholders). These participants are only counted once under each of the columns 'Total \# of participants', 'Researchers included' (if the stakeholder is a researcher), and 'Other stakeholders included'.

${ }^{c}$ Indicates focus groups in which the topic of RI education and training was explicitly discussed in depth. 
education and training policies. For a full list of topics discussed in all the focus groups, as well as the topic guide, please see https://osf.io/kdtnx/. All focus groups were conducted in English by one, two, or three facilitators (please see Table A.1 for more details) and were audio-recorded and transcribed. Participants received a comprehensive report of the focus group results (Sørensen et al. 2020).

\subsection{Participant selection and recruitment}

We used a purposive sampling strategy to identify and recruit participants from all main research areas to take part in the focus groups. We included researchers from specific disciplines and of various ranks (i.e. $\mathrm{PhD}$ students, postdoctorate researchers, and assistant, associate, and full professors). This was to allow for comparisons of views across disciplines and academic ranks, as well as to ensure that the view of stakeholders from various groups across Europe would be represented in the guidance developed by the SOPs4RI consortium. When recruiting junior researchers (e.g. PhD students), we only recruited those who had at least a few years of experience with research to ensure familiarity with the focus group topic and to minimize potential power dynamics. For half the focus groups, we also attempted to include at least two other research stakeholders with disciplinaryspecific knowledge (e.g. confidential RI counselors, funders, editors, research ethics (RE) committee members, and RI trainers). We used two strategies simultaneously to identify and recruit participants: (1) we approached contacts from our networks via email, followed by snowballing, and we (2) looked on research institutions' websites to identify and invite suitable candidates (i.e. researchers of various ranks or other stakeholders with disciplinary-specific knowledge). The composition of the focus groups can be found in Table 1 . Information on dropouts from the focus groups can be found in Table A.2.

\subsection{Data analysis}

We used a hybrid deductive-inductive thematic analysis approach to analyze the data as in Fereday and Muir-Cochrane (2006) and Swain (2018), in an iterative manner. Data analysis was conducted using the program MAXQDA 2018. In consultation with N.E. and J.T., K.L. developed a preliminary deductive code book based on a categorization of concepts related to RI or RE education contained in two scoping reviews about RI policies (Gaskell et al. 2019; Ščepanović et al. 2021). With this step, we intended to find views that can serve as a basis for guidance on RI education. The code book was subsequently added to and adjusted over five iterations collaboratively by K.L., N.E., R.R., P.K., and J.T. The development of the code book can be found here: https://osf.io/dyta8/.

The full transcripts of the eight focus groups with in-depth discussions on RI education and training were analyzed; these were supplemented by an analysis of parts of the remaining twenty-two focus group transcripts that contained data relevant for RI education and training (consisting of forty-nine pages of transcripts in total). A first round of coding was done per unit of meaning (i.e. sentence, phrase, or paragraph that refers to one code) by labeling text representing the codes from the preliminary code book. Emerging inductive codes were added to the code book and used for analysis, either as separate from the deductive codes or expanding on the deductive codes. To increase reliability, all the data were also analyzed by an independent coder in a second round of coding using the updated code book. Any discrepancies between the first coder (K.L.) and second coder (N.E., P.K., and R.R.) were discussed, in order to make a final decision and make any necessary alterations to the code book. A third coder (J.T.) was consulted in case of disagreements. No new codes emerged when coding the last focus group transcripts, indicating that data saturation was reached.

After coding was complete, we clustered the codes into overarching themes and subthemes (Fereday and Muir-Cochrane 2006). The data were initially analyzed using an across-case analysis strategy, where we looked at commonalities in the data across cases (i.e. disciplines, stakeholder types, and ranks; Ayres et al. 2003). Next, using a withincase analysis strategy, we re-examined the earlier stages of the data analysis and scrutinized the alignment between the overarching themes/subthemes with quotes within disciplines, stakeholder types (i.e. researchers versus other stakeholders), and academic ranks (i.e. junior versus senior researchers), to ensure that the interpretation of the data applies well across all cases (Ayres et al. 2003; Fereday and Muir-Cochrane 2006).

\section{Results}

Based on our analysis, we identified five overarching themes arising from the data: (1) education should be available to all involved in research; (2) the education and training approach, goals, and content should be tailored; (3) institutions should actively motivate trainees to engage in RI education and training; (4) both formal and informal educational formats should be included in the RI education and training policy; and (5) institutions should take into account various individual, institutional, and system-of-science factors when implementing RI education. Figure 1 provides a summary of the themes and subthemes. We elaborate further on each theme in the sections below. Our analysis showed few differences in perspectives among participants from different disciplines and ranks in the study; any differences are highlighted in the explanation of results for each theme. More information on the quotes found in the text, as well as additional illustrative quotes per theme, are presented in Tables A.3-A.7.

\subsection{Training availability}

Participants across disciplines stressed that RI education should be available for all researchers (Table A.3). Many mentioned that their institutions have RI training available at least for PhD students. However, some of these trainings seemed to be recently established, with multiple junior researchers remarking that they had never received RI training. This might especially be relevant for junior postdoctoral researchers, as highlighted by one postdoc's concern that 'there are so many gaps, relating specifically to the postdoc experience' (junior researcher, humanities, Netherlands). Some participants remarked that starting RI education at the $\mathrm{PhD}$ level is too late and that bachelor and master students also need to be targeted.

Many participants across disciplines emphasized that in order to ensure adequate support and supervision to junior 


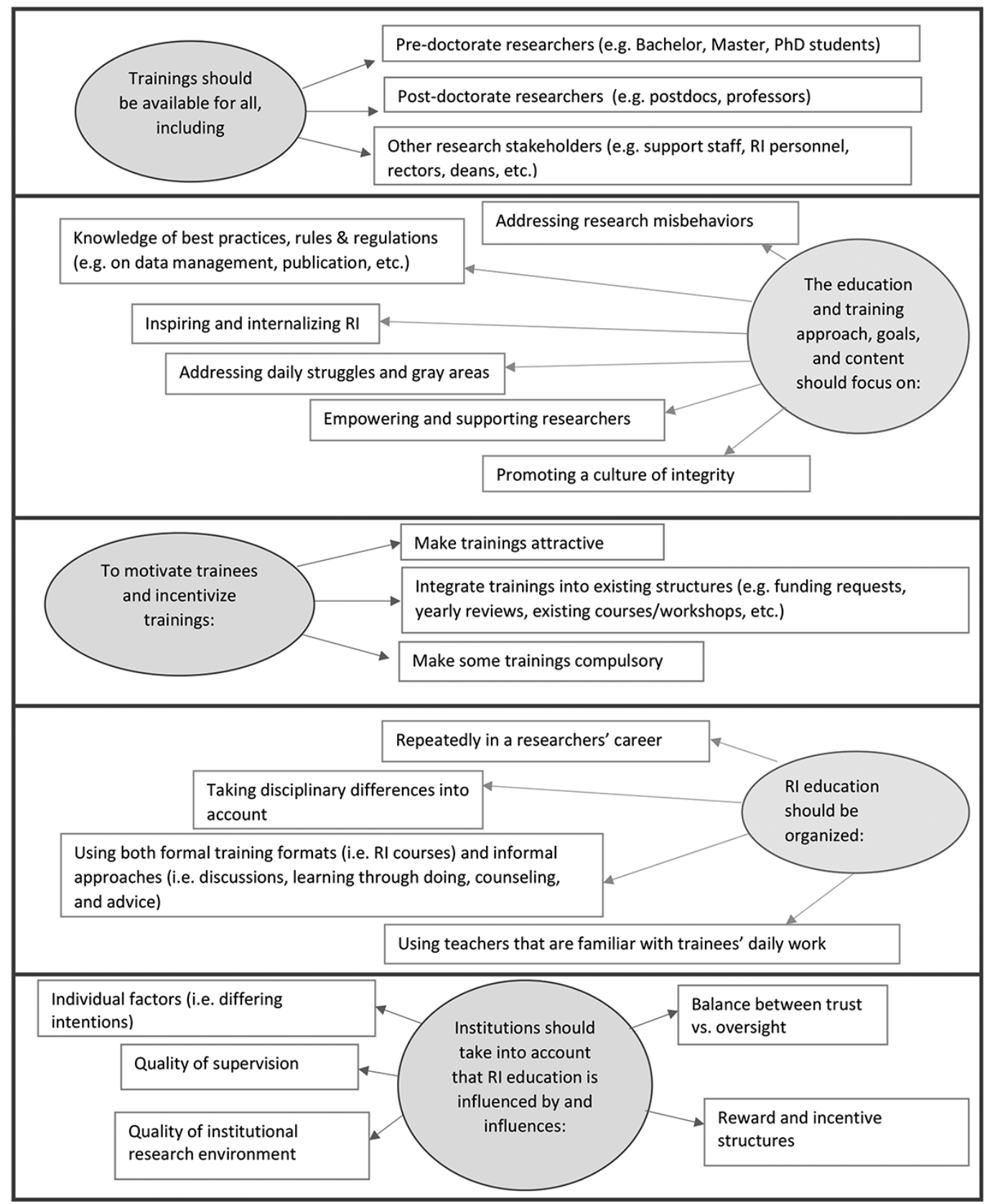

Figure 1. Main findings on participants' perceptions of RI education and training policies.

researchers, senior researchers should also be trained. Additionally, they highlighted that senior researchers also need to be trained for their own learning process, i.e. not only for the sake of supporting others. Only in one humanities focus group did participants mention that professors in their institution receive RI training. Additionally, a few researchers argued that RI training should also target other research stakeholders in the institution, including staff, managers, rectors, and deans, since they have an important responsibility regarding RI, with one participant even exclaiming that 'they are ruling' research (senior researcher, natural sciences, Croatia).

\subsection{Education and training approaches, goals, and content}

While one researcher wished that RI trainings would cover all aspects of RI, others argued that it is not feasible to teach everything and stressed that training needs to be tailored to the disciplinary field and target group. A range of-sometimes conflicting-goals, approaches, and content were described as appropriate for RI education and training (Table A.4). First, there was at least some mention of addressing research misbehaviors (such as plagiarism, fraud, and data manipulation) and even sexual misconduct during RI training in most focus groups. One researcher explained that research misbehaviors 
are often unintentional and 'many students commit plagiarism without realizing it' (senior researcher, medical sciences, Greece), so it is important to raise awareness about them by showing trainees the harmful consequences of serious and minor research misbehaviors. However, a few participants were concerned that merely telling trainees what not to do may not have sufficient impact, with one participant stating that telling researchers 'Okay, this is bad okay' will not change 'anything that drives people towards that behavior' (senior researcher, social sciences, Netherlands).

Secondly, across disciplines, there was much discussion about making participants aware of best practices, rules, and regulations relating to RI, since it is important to place-in the words of one participant-'the full responsibility [...] on the institution [...] to make sure that all its research performing employees are actually aware of current rules or changes in rules' (funder, medical sciences, Denmark). Topics that fell under these discussions varied between disciplines and included 'ethics' (humanities); open science (humanities and social sciences); conflicts of interest (humanities and medical sciences); authorship, data management, and analysis (social, medical, and natural sciences); peer review (social and medical sciences); citations (social sciences); good clinical practice, research with animals, and confidentiality, (medical sciences); and lab work (natural sciences).

The third approach to RI education discussed across disciplines was about inspiring trainees and helping them to internalize RI by engaging them in reflections on why they should engage in good research practices, as stipulated in RI guidelines. One participant was concerned that discussions about RI came about because researchers' 'professional[ism] is under threat and the public imagery is turning on its head' rather than because 'from the inside [researchers] believe in these things' (senior researcher/RI committee member, humanities, Netherlands). Many acknowledged that trainings should help trainees to reflect on their intrinsic motivations for RI. Ideas on how to do this included discussing virtues, testimonials, experiences, and quotes from inspirational figures and examples.

Fourthly, there was widespread agreement that practical considerations in research can sometimes contradict ethical and legal ones, leading to the presence of so-called 'gray areas' where the right course of action is not crystal clear. For instance, participants in several focus groups mentioned that it can be challenging to abide by best practices regarding safe and secure data management in cases where available secure data management infrastructure is not of high quality or convenient to use. It was stressed that RI education should address the real daily struggles and gray areas that researchers encounter, rather than only-as one participant remarked-'having courses that are idealizing the situation' (senior researcher, social sciences, Netherlands). Many also advocated for training that aims to empower and support researchers to deal with power dynamics, as an additional approach to RI education. One participant explained that $\mathrm{PhD}$ students are left with doing many tasks 'on their own time, really,[...] become tennis balls' (senior researcher, humanities, Netherlands) and need support to speak up in research, while another mentioned that 'just knowing what rights you could have if it was an ideal world' would be helpful (junior researcher, social sciences, Germany). Another participant even remarked that 'we can also train our students [...to] stand up for their thing', since 'without that you cannot become a good researcher' (senior researcher, social sciences, Netherlands).

Building an RI culture was considered the ultimate, overarching goal of RI education according to multiple participants from various disciplines. For instance, one participant mentioned that RI education is 'helpful [...to] change the culture, the mentality' (senior researcher, natural sciences, Croatia), while another explained that the ultimate goal of not only RI education but institutional RI policies in general is 'that there's a culture of research integrity and there are all kinds of instruments that you can think about to promote this culture', including education (senior researcher/RI committee member, humanities, Netherlands). Participants advocated for creating a research culture consisting of various features such as the presence of common standards and expectations; an erroraccepting culture that makes it possible for researchers to be open about mistakes and doubt; a culture of collaboration and data sharing; and a space for open, joint reflections. A few participants were hesitant about the feasibility of building an RI culture through training, with one remarking somewhat cynically 'I am super curious how you are going to change an existing culture with a training session' (senior researcher, natural sciences, Netherlands).

\subsection{Motivations and incentives to participate in RI training}

Resistance to RI education was one of the main challenges identified by most study participants, particularly when it comes to senior researchers (Table A.5). For instance, one participant was concerned that 'the thing with [RI] courses is that the people who need it won't do it' (senior researcher, humanities, Netherlands). Another mentioned that 'research integrity is not a favorite' even among $\mathrm{PhD}$ students (senior researcher/research director, medical sciences, Belgium). The main reasons discussed for why researchers might not be motivated to participate in RI training were the presence of competing priorities and trainings; the fact that many researchers believe that RI education is not helpful for them since they are already well-intentioned; and the perception that trainings are often not tailored enough to the specific disciplinary needs of trainees. Solutions on how to address resistance revolved around making trainings more attractive using various strategies such as 'dropping the [absolute] term' RI as it is 'religious' (senior researcher, social sciences, Netherlands) and replacing it with a more neutral one such as 'scientific conduct' (senior researcher/RI teacher, social sciences, Netherlands); tailoring training programs to the needs of the trainees; updating training to address new issues in research; focusing on specific RI topics such as data management, rather than general RI consideration; showing that trainings are effective; and making trainees curious by discussing case studies 'maybe a little bit like gossip' (privacy officer, natural sciences, Netherlands).

Participants across disciplines also advocated for integrating RI education into bachelor and master courses related to research, and courses targeted at researchers on other topics, such as academic writing, methodology, and personal development and leadership. Furthermore, there were discussions on tying RI education and training to funding and assessments in order to-as put by one participant-'use that competitiveness' of research to push for RI education ( $\mathrm{RE}$ 
committee secretary, natural sciences, Netherlands). In general, there was agreement among the medical and natural science participants that making RI training mandatory could be beneficial, with some recommending it not only for $\mathrm{PhD}$ students but also for senior researchers and policymakers. However, a few participants raised concerns that mandating RI training 'will surely create a lot of resistance' (RI officer, natural sciences, Denmark) and could even become an 'obstacle' to training (senior researcher, social sciences, Denmark). Within the humanities and social sciences, it was suggested that the decision on whether to make training mandatory depends on the type of training provided, with one participant proposing that mandatory courses are suitable when addressing 'a certain code of conduct', but courses on issues such as referencing do not 'necessarily' have 'to be mandatory' (senior researcher, humanities, Netherlands).

\subsection{Organization of RI education and design of trainings}

Participants discussed a range of issues concerning the organization of RI education and design of trainings (Table A.6). First, there was agreement across disciplines that RI education should be a continuous, even 'lifelong' (senior researcher, medical sciences, Denmark), learning process. Therefore, it was recommended to repeat training for all academic ranks every few years as illustrated by the following remark from a participant: '[Continuous training is] important to make sure that you calibrate your compass each time to make sure you can make decisions [about RI]' (senior researcher/RI teacher, social sciences, Netherlands). Secondly, there were various ideas about the format of RI education with references being made to both explicit training (i.e. 'formal' courses) and making use of implicit opportunities to educate about RI (i.e. via 'informal' educational approaches). While there was consensus among participants in the medical and natural sciences about the usefulness of formal RI courses, there was disagreement about this in the humanities and social sciences. For instance, one humanities researcher explained that formal 'training doesn't make sense' unless there is a need to address a 'legalistic framework' (senior researcher, humanities, Netherlands), while a social scientist remarked that 'not every aspect of research integrity can be trained explicitly' in a course (senior researcher, social sciences, Germany). Different types of methods were suggested as appropriate for formal courses, including interactive workshops, games, small tutorials, online training, case study discussions, peer interviews about the research process, and open discussions.

Participants in the social and natural sciences, and the humanities, also advocated for using informal discussions outside of formal courses to educate about RI, with one explaining that much can be learned about RI during conversations 'over coffee time' (senior researcher, RI committee member, humanities, Netherlands). There were also ideas about specific situations that can facilitate such informal discussion opportunities, including institute and team meetings, hackathons, mistake cakes (i.e. events where researchers openly discuss mistakes they have done in research over cake), and journal clubs. Similarly, it was highlighted that much of RI education happens implicitly while doing research, applying for ethics review, receiving supervision, and being socialized in a particular research environment; one participant referred to this as 'learning by doing' (senior researcher, social sciences, Germany). It was thought that RI education policies should exploit this implicit type of informal learning. Participants mentioned that as particular questions about RI come up in practice, researchers need access and referral to resources and guidance documents on good research practice to consult in order to address their questions and further their RI education. Besides this, the need for researchers to have access to people that can provide face-to-face advice on doubts that come up in research was also discussed. Both specialized advisors such as librarians, privacy officers, and ethics committee members, and general RI advisors were referred to. However, there was some concern raised that RI advisors are often perceived as too formal and related to procedures of reporting misconduct to be deemed approachable by researchers for day-to-day questions on RI, with one participant asking 'So why would you take that step [of approaching one]?' (senior researcher/RI teacher, social sciences, Netherlands).

There was a diversity of opinions offered on the issue of who is suitable to provide RI trainings. One humanities participant suggested asking existing experts to allocate time for providing RI training rather than hiring new people, while another mentioned that teachers should have been themselves taught by 'some sort of authority' on how to teach RI (junior researcher, humanities, Croatia). Others emphasized the need to hire RI trainers with discipline-specific knowledge, with one participant mentioning that that is necessary to ensure that the trainer 'really is aware of what they're talking about' (senior researcher, humanities, Netherlands). While it was thought that trainers should have experience with research, there was disagreement among participants about whether researchers would be motivated to provide RI trainings. However, most agreed that trainings should be provided in a discipline-specific context (e.g. by doctoral schools or faculties). It was mentioned that informal educational events (e.g. RI discussions during journal clubs) could address common RI issues faced by multiple disciplines. Furthermore, there were different ideas on whether trainings should be provided in full courses of smaller workshops or integrated in other courses, with a few suggestions across disciplines that decisions on this should be based on the target group of the training, and the institutional resources and capacities available.

\subsection{Factors influencing the implementation of RI education and training policies}

The focus group participants identified five factors that might influence the implementation of institutional RI education and training policies: (1) individual factors of trainees, (2) supervision, (3) institutional research environment, (4) trust versus oversight, and (5) reward and incentive structures (Table A.7). Various facilitators and barriers were mentioned for each factor, and these are further elaborated on in this section. Please also take into consideration that these factors are interrelated and likely influence one another. To begin with, one participant expressed that 'the individual is also important and that's sometimes very difficult to change' via training (senior researcher/research director, medical sciences, Belgium). This view was mirrored by others who warned about a small minority of researchers who are ill-intentioned and will engage in misconduct to cut corners; trainings will likely not be able to change these attitudes. Others highlighted that 
cultural differences among individual researchers can lead to a difference in understanding of research norms and values.

Regarding mentorship, participants across disciplines highlighted that unless trainees' supervisors support them in engaging in responsible research practice, RI training might be futile. For instance, one remarked that 'we train the $\mathrm{PhD}$ students in these courses for half a day or one day during our years, when they get trained by that professor [...] for four years' (senior researcher, natural sciences, Netherlands), while another explained that 'If you're supervised in a correct and responsible way you will behave automatically as you should' (junior researcher, social sciences, Germany). However, it was acknowledged that the relation between RI training and supervision is mutual, and training targeted at supervisors could improve supervision.

Similarly, across disciplines, it was mentioned that the institutional research environment and RI education have a mutual influence on each other. On the one hand, when aimed at creating a responsible research culture, RI education can support the institutional research environment. On the other hand, the research environment influences whether values and practices addressed in RI education and training are actually internalized by trainees. For instance, one participant explained that researchers are mainly taught by being socialized in their research environment since 'it's labs that train the students' (senior researcher, natural sciences, Spain). Characteristics of the research environment that could negatively influence RI education mentioned included the 'vanity' of the 'discourse of excellence' present in academia, i.e. the urge to present oneself as 'excellent' (senior researcher, humanities, Netherlands); too much competition; a rigid hierarchy; a culture of blaming; and a lack of institutional commitment to RI, e.g. in terms of data management support and bylaws.

Maintaining a balance between trust in researchers and oversight of RI in the institution was another factor that was emphasized as important across focus groups. A few preferred to have some oversight on RI in the form of rules and documents, with one researcher remarking that 'although it looks like a bureaucracy, the key is in the documents. If you don't have documents you have nothing' (senior researcher, medical sciences, Croatia). Alternatively, many others explained that there is currently too much emphasis on oversight and bureaucracy in research, which can be counterproductive. For instance, one participant mentioned that ${ }^{6}$ [increased oversight] creates more distrust towards[...]researchers instead of the thing you want to achieve[which] is to have responsible researchers' (privacy officer, natural sciences, Netherlands). Participants thought that RI education can be a means to reduce oversight; as institutions train researchers to be responsible, trust in researchers can increase, leading to reduced monitoring and oversight. However, a few humanities and social science researchers were concerned that-as put by one participant-'requiring courses is part of a general suspicion against people working here' (senior researcher, humanities, Spain), and also constitutes oversight and added bureaucracy.

Finally, there was agreement across disciplines that a major hurdle, which institutions face when providing RI education and training, is the current structure of rewards and incentives in science. One participant argued that '[if] the structures are not good then you can train as long as you like, but you're never going to change what is going to happen really, in the grassroots' (senior researcher/confidential counselor, humanities, Netherlands). Another participant explained that providing RI education and training does not address the root cause of RI problems by saying that 'it's not a lack [of awareness] of guidelines, but the way[...] that researchers are rewarded or not rewarded by funding[...] that causes most of the problems' (senior researcher, medical sciences, Belgium). Particular elements of the current reward and incentive structures that were identified as a barrier for RI included the culture of deliverables, publication pressure, precarious working conditions, and project-based funding. Interestingly, two participants (a senior researcher and funder) remarked that the competitive nature of existing reward and incentive structures is not a barrier' for RI training (natural sciences, Netherlands), since training can be embedded into these structures (e.g. if seen as a competitive advantage in grant applications). Furthermore, there was agreement that junior researchers are particularly vulnerable to these structures due to their dependence on seniors for funding.

\section{Discussion}

In this study, we assume that in order to foster RI through education, institutions need to develop and implement an overarching strategy for RI education and training, which takes into account research stakeholders' preferences and perspectives. Our results highlight that RI education is unlikely to be successful if implemented without sufficient attention to other institutional RI responsibilities (e.g. ensuring responsible supervision and a culture conducive to RI). Furthermore, our results show wide stakeholder support for RI education targeting all research stakeholders (e.g. researchers and deans; Evans et al. 2018; Fanelli 2019). The RI education and training goals, approaches, and content discussed by the participants in our study reflect the multitude of existing approaches used in training programs (Fanelli 2019; Abdi et al. 2021), suggesting that it might not be necessary or desirable to agree on the same learning goal for all educational programs. Instead, a tailored approach to RI education might be needed to sufficiently take into account disciplinary and country differences. Additionally, our results emphasize the importance of institutional efforts in motivating participants to actively take part in RI education. This is particularly important, considering the results indicating that RI education requires a continuous learning process consisting of formal and informal formats (e.g. both formal RI courses and informal discussions about RI).

A critical finding of our study is that the implementation of successful RI education is highly dependent on various individual, institutional, and system-of-science factors. These results confirm previous studies that suggest that cultural differences among individuals (Antes et al. 2018), supervision (Alfredo and Hart 2011; McGee et al. 2014), the institutional research environment (Kalichman 2007; Alfredo and Hart 2011; National Academies of Sciences, Engineering, and Medicine 2017; Satalkar and Shaw 2018), and reward and incentive structures in the system-of-science (Marie-Claude 2007; Asai et al. 2016) play a crucial role in the uptake and delivery of RI education. This highlights that addressing RI education policies in a vacuum-i.e. solely implementing RI education without addressing other institutional 
responsibilities for RI-will not be a sufficient strategy for institutions to foster RI, as they will need to develop a comprehensive institutional RI plan including policies on multiple RI topics, including supervision and the research environment, simultaneously (Mejlgaard et al. 2020). This recommendation is in line with literature suggesting that RI education should not be the mere means of fostering RI, as researchers' behavior is highly dependent on other RI factors such as promotions and evaluations (Aubert Bonn and Bouter 2021; Aubert Bonn and Pinxten 2021). Instead, institutions should create policies focusing on creating a collaborative, error-accepting, and open research environment, as well as on reducing unnecessary bureaucracies and potentially corrupting influences of hyper-competition in research. When integrated into the research endeavor-including the socialization into the research process-sufficiently, RI education could also be used as a tool to improving the research environment, e.g. by increasing awareness and reflection on RI in a research group (National Academies of Sciences, Engineering, and Medicine 2017; Labib et al. 2021).

As the results show, a variety of goals can be used for RI education and training; this highlights the difficulty of standardizing RI education and training approaches across institutions and disciplines. The majority of the goals and approaches discussed in our focus groups, including focusing on misconduct, improving knowledge of RI, internalizing RI values, addressing gray areas of research, and culture building, have also been mentioned in previous literature (DuBois 2004; Powell et al. 2007; Geller et al. 2010; Zeng and Resnik 2010; Kalichman 2014; Fanelli 2019; Valkenburg et al. 2021). An additional goal that our participants highlighted is empowering researchers, i.e. equipping researchers to deal with power dynamics and the rigid hierarchies present in academia (e.g. by giving them relevant tools and knowledge necessary to speak up about RI to their supervisors). Although the literature suggests moving away from educational approaches that solely focus on cases of research misconduct and knowledge of RI concepts toward more aspirational and cultural approaches (DuBois 2004; Kalichman 2014), there was no clear preference for a specific approach across our focus groups. It was suggested that different approaches are suitable depending on the context and target group of the training provided. For instance, the goal of empowering trainees could be considered more suitable for training targeted at junior, rather than senior researchers. Consequently, it might be most appropriate to tailor RI education and training approaches, goals, and content to the specific context and target group at hand (Watts et al. 2017). This view contrasts with pleas to agree on a fixed set of RI educational goals in order to allow for evaluations of training effectiveness (Kalichman 2007) and might explain why such agreement has not already been achieved (Fanelli 2019).

Our finding that RI training appears to be unappealing to researchers stresses the need for RI education and training policies to address training attractiveness and incentives. While multiple ways to incentivize the participation of junior researchers have previously been identified (e.g. digital badges and free meals; Fanelli 2019), our results show that it is senior researchers that need strong incentives to participate. Although it has been recognized that incentivizing senior researchers remains a challenge (Fanelli 2019), the participants in our study provided some concrete suggestions on how to address this, including ensuring that the training itself appears attractive (e.g. by addressing the real needs of the trainees), as well as integrating RI education in existing events, funding schemes, and assessments. Importantly, our results suggest that efforts to make RI education more appealing to researchers will also need to address researchers' resistance to the potential increased oversight that RI policies might introduce (Sørensen et al. 2020). What could help to prevent researchers from perceiving RI education as an additional bureaucratic hurdle is to regularly use implicit educational formats (e.g. open discussions at department meetings) to help researchers reflect on RI informally, rather than always relying on formal courses (Kalichman 2007; OECD Global Science Forum 2007; Alfredo and Hart 2011; Satalkar and Shaw 2018).

In line with existing recommendations (Forsberg et al. 2018; Ravn and Sørensen 2021), our results show that RI trainings should be provided in a disciplinary-specific context to ensure sensitivity to the real needs of trainees. Beyond this, we did not find many disciplinary differences in participants' views on important considerations for RI education and training policies. One potentially notable difference observed was that unlike participants in the medical and natural science focus groups, some humanities and social science researchers were skeptical about making RI training events compulsory. Furthermore, there was more hesitation among humanities and social science researchers than those in the medical and natural sciences, about the need for explicit and formal RI training events. These differences might be explained by the lower level of existing research regulation in the humanities and some social science fields, compared to research performed in the medical and natural sciences (Wells et al. 2014; Haven et al. 2019). The extent to which researchers are already accustomed to having their research regulated could potentially influence their acceptance of new mandatory formal courses.

\subsection{Strengths and limitations of the study}

Although there are some existing insights about research stakeholders' perceptions of RI education (ENERI 2017; Evans et al. 2018), to our knowledge, this is the largest study providing an in-depth and explicit analysis of researchers' and other relevant stakeholders' preferences regarding the development and implementation of RI education in research institutions across Europe. The results of the eight focus groups explicitly addressing the topic of RI education and training in depth were further validated based on the data from the remaining twenty-two focus groups. The large amount of data collected allowed us to make comparisons across disciplines and ranks. The data also made it clear that there are few differences across disciplines and ranks. Since not all the focus groups explicitly addressed the topic RI education and training, we did not have sufficient data to be able to make cross-country comparisons, however.

Our analysis method allowed us to link insights gained in this study to existing knowledge about RI education. Since multiple topics, including RI education, were discussed throughout the thirty focus groups, we obtained a wide breadth of data on RI during the study, but that required some sacrifices regarding depth and richness of data on specific issues. Our qualitative approach enabled us to provide 
an additional angle to discussions about RI education, which are currently dominated by considerations of training effectiveness (Fanelli 2019). Our choice to only include junior researchers with a number of years of research experience led to the inclusion of a much smaller number of junior researchers as compared to senior researchers. This means that the perspective of junior researchers, who represent a significant proportion of the research workforce, might not be sufficiently explored. Considering that previous studies have shown differences in junior and senior perceptions of RI (e.g. juniors perceive their existing research climate more negatively; Haven et al. 2019, 2020), it would be valuable to validate our findings with other junior researchers.

\section{Conclusions}

In this study, we present insights about research stakeholders' views and experiences regarding RI education, which are relevant for developing and implementing RI education and training policies. We show that researchers and other research stakeholders across disciplines in Europe recommend RI education and training policies that (1) provide RI education to all involved in research; (2) use training approaches, goals, and content tailored to the target group; (3) focus on motivating trainees; (4) include formal and informal training formats; and (5) take into account various individual, institutional, and system-of-science factors when implementing RI education. These results confirm the need for research institutions to develop a comprehensive RI plan that integrates RI education into the research endeavor (National Academies of Sciences, Engineering, and Medicine 2017; Mejlgaard et al. 2020), includes an overarching strategy on how to develop and implement RI education that is engaging for all involved in research, and tailors training to disciplinary-specific contexts.

\section{Funding}

This work was supported by the European Commission HORIZON 2020 framework program for Research and Innovation [Grant Agreement No 824481].

Conflict of interest statement. None declared.

\section{Acknowledgements}

We would like to thank all of the participants in this study for their valuable insights and time, as well as the various other people who helped us with organizing and setting up the focus groups. We would especially like to thank the following people from the SOPs4RI project, who were involved in planning, conducting, and/or transcribing the focus groups: AnnaKathrine Bendtsen, Vicko Tomić, Vasileios Markakis, Dan Gibson, Jonathan Bening, Ana Marušić, Josephine Bergmans, Eleni Spyrakou, and Giuseppe Veltri.

\section{Authors' contributions}

K.L. contributed to the design of the focus group study and was responsible for conducting focus groups, drafting and revising the data analysis protocol, analyzing the data, and drafting and revising the manuscript. N.E. contributed to revising the data analysis protocol, analyzing the data, drafting and revising the manuscript, and supervision. A.R.E., W.K., R.R., I.B., and P.K. conducted focus groups and contributed to revising the manuscript; R.R. and P.K. were also involved in the data analysis. T.R. and M.P.S. were responsible for the design of the focus group study, conducted focus groups, and contributed to revising the manuscript. G.W. and L.B. contributed to revising the data analysis protocol and the manuscript, and G.W. was also involved in conducting the focus groups. C.C. contributed to revising the manuscript. J.T. was involved in conducting and designing the focus groups, revising the data analysis protocol, drafting and revising the manuscript, and supervision.

\section{References}

(2019) 'Research Integrity is Much More than Misconduct', Nature, 570: 7.

Abdi, S., Pizzolato, D., Nemery, B., et al. (2021) 'Educating PhD Students in Research Integrity in Europe', Science and Engineering Ethics, 27: 1-19.

Alexander, M. and Williams, W. (2008) A Guidebook for Teaching Selected Responsible Conduct of Research Topics to A Culturally Diverse Trainee Group., 1st edn. Rockville: The Children's Hospital of Philadelphia.

Alfredo, K. and Hart, H. (2011) 'The University and the Responsible Conduct of Research: Who Is Responsible for What?' Science and Engineering Ethics, 17: 447-57.

Anderson, M. S., Horn, A. S., Risbey, K. R., et al. (2007) 'What Do Mentoring and Training in the Responsible Conduct of Research Have to Do with Scientists' Misbehavior? Findings from a National Survey of NIH-funded Scientists', Academic Medicine, 82: 853-60.

Antes, A. L., English, T., Baldwin, K. A., et al. (2018) 'The Role of Culture and Acculturation in Researchers' Perceptions of Rules in Science', Science and Engineering Ethics, 24: 361-91.

Antes, A. L., Murphy, S. T., Waples, E. P., et al. (2009) 'A Metaanalysis of Ethics Instruction Effectiveness in the Sciences', Ethics \& Behavior, 19: 379-402.

Asai, A., Okita, T., and Enzo, A. (2016) 'Conflicting Messages Concerning Current Strategies against Research Misconduct in Japan: A Call for Ethical Spontaneity', Journal of Medical Ethics, 42: 524-7.

Aubert Bonn, N. and Bouter, L. (2021) 'Research Assessments Should Recognize Responsible Research Practices-Narrative Review of a Lively Debate and Promising Developments'. 10.31222/osf.io/82rmj.

Aubert Bonn, N. and Pinxten, W. (2021) 'Rethinking Success, Integrity, and Culture in Research (Part 2)-A Multi-actor Qualitative Study on Problems of Science', Research Integrity and Peer Review, 6: $1-18$.

Ayres, L., Kavanaugh, K., and Knafl, K. A. (2003) 'Within-case and Across-case Approaches to Qualitative Data Analysis', Qualitative Health Research, 13: 871-83.

Boehme, O., Föger, N., Hiney, M., et al. (2016) 'Research Integrity Practices in Science Europe Member Organisations', Science Europe, 1-52.

DuBois, J. M. (2004) 'Is Compliance a Professional Virtue of Researchers? Reflections on Promoting the Responsible Conduct of Research', Ethics \& Behavior, 14: 383-95.

ENERI. (2017), Report of Stakeholder/Focus Group Workshop in Athens. <https://eneri.eu/wp-content/uploads/2018/06/ReportStakeholder-Workshop-Athens.pdf > accessed 13 Oct 2021.

Evans, N., Veldkamp, C., Valentini, E., et al. (2018), EnTIRE Stakeholder Consultation Report. <https://ec.europa.eu/research/ participants/documents/downloadPublic?documentIds $=080166 \mathrm{e} 5$ be1fb1a0\&appId=PPGMS $>$ accessed 13 Oct 2021. 
Fanelli, D. (2019), MLE on Research Integrity - Training \& Education - Thematic Report No 4. <https://rio.jrc.ec.europa.eu/sites/ default/files/report/MLE\%20RI\%20No4\%20-\%20Training\%20 and $\% 20$ education.pdf $>$ accessed 13 Oct 2021.

Fereday, J. and Muir-Cochrane, E. (2006) 'Demonstrating Rigor Using Thematic Analysis: A Hybrid Approach of Inductive and Deductive Coding and Theme Development', International Journal of Qualitative Methods, 5: 80-92.

Forsberg, E.-M., Anthun, F. O., Bailey, S., et al. (2018) 'Working with Research integrity-Guidance for Research Performing Organisations: The Bonn PRINTEGER Statement', Science and Engineering Ethics, 24: 1023-34.

Gaskell, G., Ščepanović, R., Buljan, R., et al. (2019), D3.2: Scoping Reviews Including Multi-level Model of Research Cultures and Research Conduct. <https://www.sops4ri.eu/wp-content/uploads/ D3.2_Scoping-reviews-including-multi-level-model-of-research-cult ures-and-research-conduct.pdf> accessed 13 Oct 2021.

Geller, G., Boyce, A., Ford, D. E., et al. (2010) 'Beyond "Compliance": The Role of Institutional Culture in Promoting Research Integrity', Academic Medicine, 85: 1296-302.

Godecharle, S., Nemery, B., and Dierickx, K. (2014) 'Heterogeneity in European Research Integrity Guidance: Relying on Values or Norms?' Journal of Empirical Research on Human Research Ethics, 9: 79-90.

Haven, T., Pasman, H. R., Widdershoven, G., et al. (2020) 'Researchers' Perceptions of A Responsible Research Climate: A Multi Focus Group Study', Science and Engineering Ethics, 26: 3017-36.

Haven, T., Tijdink, J. K., Martinson, B. C., et al. (2019) 'Perceptions of Research Integrity Climate Differ between Academic Ranks and Disciplinary Fields: Results from a Survey among Academic Researchers in Amsterdam', PLoS One, 14: e0210599.

Hermerén, G., Zwart, H., Marušić, A., et al. (2019), MLE on Research Integrity - Final Report. <https://rio.jrc.ec.europa. eu/sites/default/files/report/MLE\%20RI_Final\%20Report_0.pdf> accessed 13 Oct 2021

Joynson, C. and Leyser, O. (2015) 'The Culture of Scientific Research', F1000Research, 4: 1-11.

Kalichman, M. (2007) 'Responding to Challenges in Educating for the Responsible Conduct of Research', Academic Medicine, 82: 870-5. (2013) 'A Brief History of RCR Education', Accountability in Research, 20: 380-94.

(2014) 'Rescuing Responsible Conduct of Research (RCR) Education', Accountability in Research, 21: 68-83.

Labib, K., Roje, R., Bouter, L., et al. (2021) 'Important Topics for Fostering Research Integrity by Research Performing and Research Funding Organizations: A Delphi Consensus Study', Science and Engineering Ethics, 27: 1-22.

Marie-Claude, R. (2007) 'Who Is Responsible? Supervisors and Institutions Need to Focus on Training in the Responsible Conduct of Research and Change the Culture in the Laboratory', EMBO Reports, 8: 706.

Marusic, A., Wager, E., Utrobicic, A., et al. (2016) 'Interventions to Prevent Misconduct and Promote Integrity in Research and Publication', Cochrane Database of Systematic Reviews, 4: MR000038.

Masadeh, M. (2012) 'Training, Education, Development and Learning: What Is the Difference?' European Scientific Journal, 8: 62-8.

McGee, R., Schneeweis, D. M., Hitsman, B., et al. (2014) 'Beyond Responsible Conduct: Taking Responsibility for the Responsible Conduct of Others', Journal of Microbiology \& Biology Education, 15: 96.

Mejlgaard, N., Bouter, L. M., Gaskell, G., et al. (2020) 'Research Integrity: Nine Ways to Move from Talk to Walk', Nature, 586: 358-60.

National Academies of Sciences, Engineering, and Medicine. (2017) 'Education for the Responsible Conduct of Research'. In National
Academies of Sciences, Engineering, and Medicine (ed.): Fostering Integrity in Research, pp. 195-204. Washington, DC: National Academies Press.

OECD Global Science Forum. (2007), Best Practices for Ensuring Scientific Integrity and Preventing Misconduct. <http://www. oecd.org/science/inno/40188303.pdf> accessed 13 Oct 2021.

Pizzolato, D., Abdi, S., and Dierickx, K. (2020) 'Collecting and Characterizing Existing and Freely Accessible Research Integrity Educational Resources', Accountability in Research, 27: 195-211.

Pizzolato, D. and Dierickx, K. (2021) 'Stakeholders' Perspectives on Research Integrity Training Practices: A Qualitative Study', BMC Medical Ethics, 22: 1-13.

Powell, S. T., Allison, M. A., and Kalichman, M. W. (2007) 'Effectiveness of A Responsible Conduct of Research Course: A Preliminary Study', Science and Engineering Ethics, 13: 249-64.

Ravn, T. and Sørensen, M. P. (2021) 'Exploring the Gray Area: Similarities and Differences in Questionable Research Practices (Qrps) across Main Areas of Research', Science and Engineering Ethics, 27: $1-33$.

Resnik, D. B. and Shamoo, A. E. (2011) 'The Singapore Statement on Research Integrity', Accountability in Research, 18: 71-5.

Rifai, N., Annesley, T. M., Moore, S., et al. (2019) 'Maintaining Research and Publication Integrity', Clinical Chemistry, 65: 230-5.

Satalkar, P. and Shaw, D. (2018) 'Is Failure to Raise Concerns about Misconduct a Breach of Integrity? Researchers' Reflections on Reporting Misconduct', Accountability in Research, 25: 311-39.

Ščepanović, R., Labib, K., Buljan, I., et al. (2021) 'Practices for Research Integrity Promotion in Research Performing Organisations and Research Funding Organisations: A Scoping Review', Science and Engineering Ethics, 27: 1-20.

Science Europe. (2017), Advancing Research Integrity Practices and Policies: From Recommendation to Implementation. Brussels. $<$ https://www.scienceeurope.org/media/lfdme4ev/ws_report_integri ty_practices_policies.pdf> accessed 13 Oct 2021.

SOPs4RI. (2020), Guideline for Promoting Research Integrity in Research Performing Organisations. <https://ec.europa.eu/ info/funding-tenders/opportunities/docs/2021-2027/horizon/guida nce/guideline-for-promoting-research-integrity-in-research-perform ing-organisations_horizon_en.pdf $>$ accessed 13 Oct 2021.

Sørensen, M., Ravn, T., Bendtsen, A., et al. (2020), D5.2: Report on the Results of the Focus Group Interviews. <https://sops4ri.eu/wp-cont ent/uploads/D5.2_Report-on-the-Results-of-the-Focus-Group-Inte rviews-1.pdf> accessed 13 Oct 2021.

Sørensen, M. P., Ravn, T., Marušić, A., et al. (2021) 'Strengthening Research Integrity: Which Topic Areas Should Organisations Focus On?' Humanities and Social Sciences Communications, 8: $1-15$.

Swain, J. (2018) A Hybrid Approach to Thematic Analysis in Qualitative Research: Using A Practical Example. London: Sage Publications Ltd.

Titus, S. and Bosch, X. (2010) 'Tie Funding to Research Integrity', Nature, 466: 436-7.

Valkenburg, G., Dix, G., Tijdink, J., et al. (2021) 'Expanding Research Integrity: A Cultural-Practice Perspective', Science and Engineering Ethics, 27: 1-23.

Watts, L. L., Medeiros, K. E., Mulhearn, T. J., et al. (2017) 'Are Ethics Training Programs Improving? A Meta-analytic Review of Past and Present Ethics Instruction in the Sciences', Ethics \& Behavior, 27: 351-84.

Wells, J. A., Thrush, C. R., Martinson, B. C., et al. (2014) 'Survey of Organizational Research Climates in Three Research Intensive, Doctoral Granting Universities', Journal of Empirical Research on Human Research Ethics, 9: 72-88.

Zeng, W. and Resnik, D. (2010) 'Research Integrity in China: Problems and Prospects', Developing World Bioethics, 10: 164-71. 
Table A.1. Characteristics of focus group (FG) facilitators and observers.

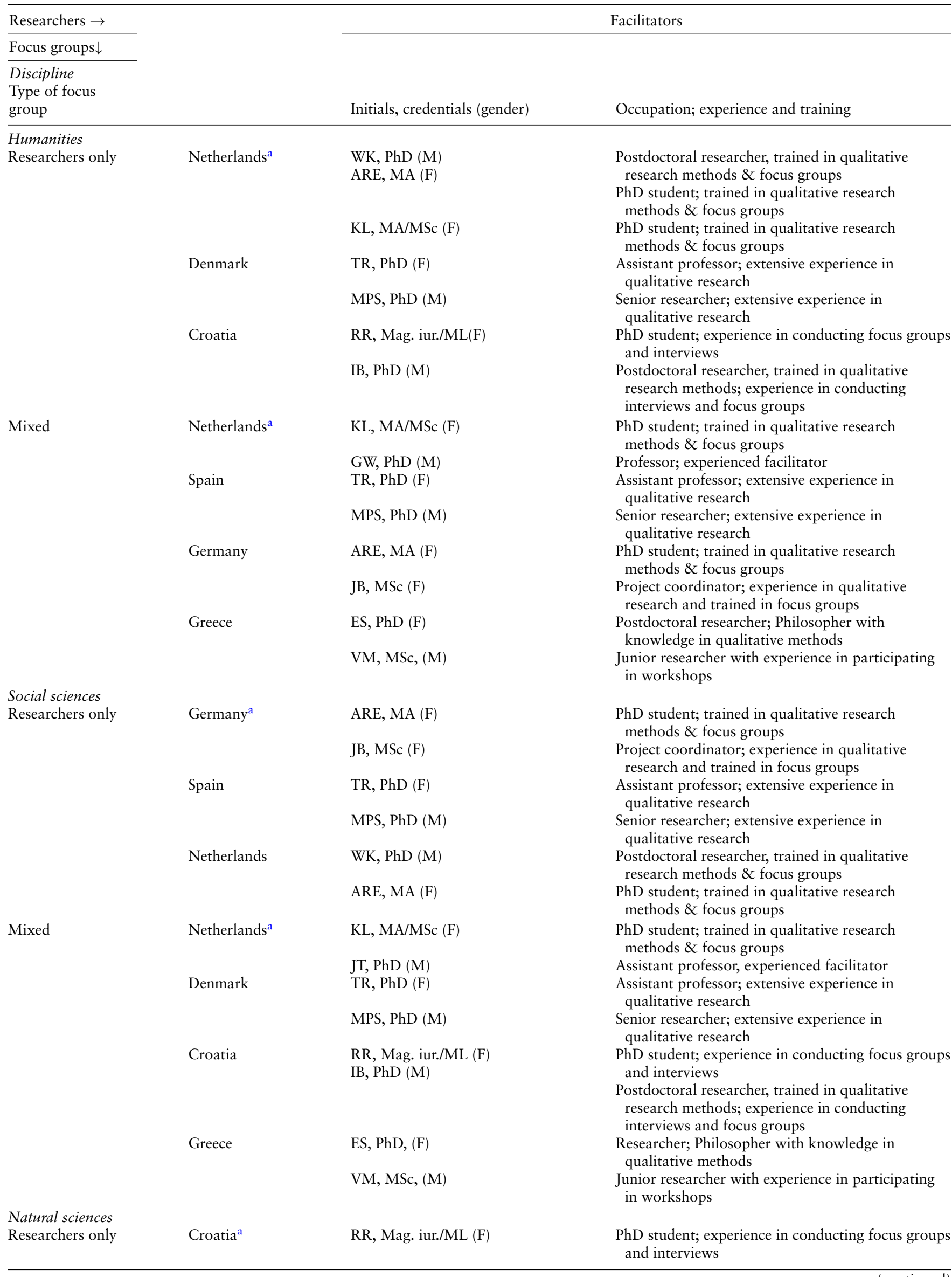


Table A.1. (Continued)

\begin{tabular}{l} 
Researchers $\rightarrow$ \\
\hline Focus groups $\downarrow$ \\
\hline Discipline \\
Type of focus \\
group
\end{tabular}

Facilitators

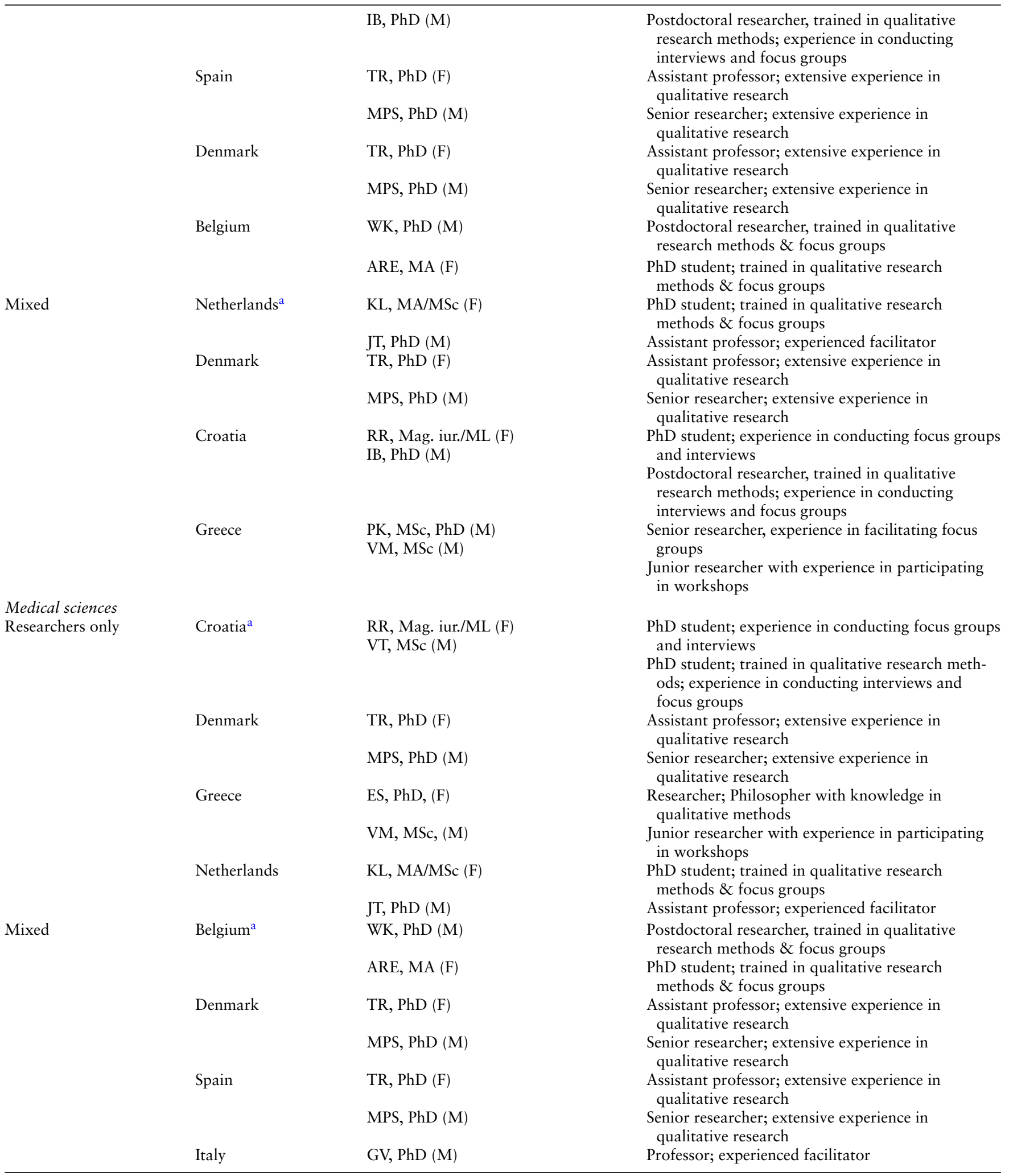

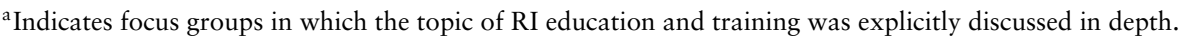


Table A.2. Information on dropouts from focus groups (FGs).

\begin{tabular}{|c|c|c|c|c|}
\hline Focus groups $\downarrow$ & & & & \\
\hline $\begin{array}{l}\text { Discipline } \\
\text { Type of focus group }\end{array}$ & & Number of dropouts & $\begin{array}{l}\text { Junior/senior researcher or } \\
\text { other stakeholder? }\end{array}$ & Reasons for dropout \\
\hline Researchers only & Netherlands ${ }^{a}$ & 0 & - & - \\
\hline & Denmark & 2 & Senior researchers & Personal circumstances \\
\hline & Croatia & 0 & & \\
\hline Mixed & Netherlands ${ }^{a}$ & 1 & Senior researcher & Personal circumstances \\
\hline & Greece & 1 & Senior researcher & Competing obligation \\
\hline Social sciences & & & & \\
\hline Researchers only & Germany $^{\mathrm{a}}$ & 0 & - & - \\
\hline & Spain & 2 & Senior researchers & $\begin{array}{l}\text { Urgent competing } \\
\text { obligations }\end{array}$ \\
\hline & & & & Change of mind \\
\hline & & & Other stakeholder & Competing obligations \\
\hline & Croatia & 2 & Junior researcher & Other obligations \\
\hline & & & Other stakeholder & \\
\hline & $\begin{array}{l}\text { Greece (conducted } \\
\text { online) }\end{array}$ & $3^{c}$ & Senior researchers & Competing obligations \\
\hline Natural sciences & & & & \\
\hline Researchers only & Croatia $^{a}$ & 0 & & \\
\hline & Spain (conducted online) & 4 & $\begin{array}{l}1 \text { Senior researcher } \\
3 \text { Junior researchers }\end{array}$ & $\begin{array}{l}\text { COVID-19 lockdown- } \\
\text { related scheduling } \\
\text { problems }\end{array}$ \\
\hline & Denmark & 0 & - & - \\
\hline & $\begin{array}{l}\text { Belgium (conducted } \\
\text { online) }\end{array}$ & 0 & - & - \\
\hline & $\begin{array}{l}\text { Denmark (conducted } \\
\text { online) }\end{array}$ & 3 & $\begin{array}{l}1 \text { Other stakeholder } \\
2 \text { Senior researchers }\end{array}$ & $\begin{array}{l}\text { COVID-19 lockdown- } \\
\text { related scheduling } \\
\text { problems }\end{array}$ \\
\hline & Greece & 2 & Senior researchers & $\begin{array}{l}\text { Unknown; } \\
\text { Competing obligation }\end{array}$ \\
\hline & Netherlands & 0 & - & - \\
\hline Mixed & $\begin{array}{l}\text { Belgium }^{\mathrm{a}} \text { (conducted } \\
\text { online) }\end{array}$ & 1 & Senior researcher & Competing obligations \\
\hline & $\begin{array}{l}\text { Denmark (conducted } \\
\text { online) }\end{array}$ & 2 & $\begin{array}{l}\text { Senior researcher Other } \\
\text { stakeholder }\end{array}$ & $\begin{array}{l}\text { Unknown (no show) } \\
\text { Personal circumstances }\end{array}$ \\
\hline & Spain (conducted online) & 2 & $\begin{array}{l}\text { Junior researcher } \\
\text { Other stakeholder }\end{array}$ & COVID-19 lockdown \\
\hline & Italy (conducted online) & 0 & - & - \\
\hline
\end{tabular}

${ }^{a}$ Indicates focus groups in which the topic of RI education and training was explicitly discussed in depth.

${ }^{b}$ We had originally invited five additional people to this focus group (three senior researchers, two junior researchers), but due to the high number of participants, we had to cancel their participation.

${ }^{c}$ The original focus group took place live (i.e. in a face-to-face setting) and included five participants. However, the recording was stolen, together with the hard disk of the secure server of the laboratory, where the files were kept. The researchers in Greece (P.K. and C.C.) notified the researchers responsible for the study design and ethics approval (T.R. and M.S.), according to the privacy policy of the focus group study. The focus group was repeated via online means, but only two out of the original five interviewees participated. 
Table A.3. Example quotes about the theme 'Training availability'.

Theme/subtheme Quotes

Training availability

At the pre-doctorate level

At the post-doctorate level

For other stakeholders
'And what's important is that absolutely everyone is to take that training, it's not just the junior.'-Senior researcher, humanities, Netherlands

'But if I teach PhD students and I tell them this is how to do things right, this is how to do things in [a responsible] way, then they go back to the labs where the norms are different and where they have to sort of fight against supervisors who are doing things the way they've always done them, which is fine because they just learnt it that way. But now there are new insights, new cultures to create. But then the PhD students have to do all the work. So I think it's very important when you think about education to make sure you include all levels.'-Senior researcher/RI teacher, social sciences, Netherlands

'I think it's important that mainly at the start of that career as a scientist, it's important to have such a training. Of course also later on it's important, but I think doctoral school is a good place to do that.'-Senior researcher/Research director, medical sciences, Belgium

'And I think it's, it would be good and helpful to start with that even on graduate or undergraduate level. Maybe not just like full course, but maybe part of some lectures. And then on the PhD level and, it would be definitely, it should be whole course, yeah.'-Junior researcher, natural sciences, Croatia

'There were no systematic trainings on good, scientific practices when I started. Up to now, there was no training in our research institute, also the university I studied my PhD.'-Junior researcher, social sciences, Croatia

'So I did [an RI] course last year. It's actually made, for I think first year PhD students but it wasn't designed when I was a first year PhD student.'-Junior researcher, social sciences, Netherlands

'There are so many gaps, relating specifically to the postdoc experience because..that I go to staff meetings but $85 \%$ of what is said, is not something I can do anything about or with... I don't go to the PhD meetings because there all about the $\mathrm{PhD}$ experience, which I've already gone through..If anybody is coming to do research in any capacity they should be folded into this training perspective.'-Junior researcher, humanities, Netherlands

'Senior researcher 1: I think earlier studies indicated that the most difficult part, or where do things go most easily wrong have to do with $\mathrm{PhD}$ supervisee relationship, so some sort of training on the part of the university to train university professors on $\mathrm{PhD}$ supervision.

Senior researcher 2: With this, I have that actually, once a year I think, or twice a year they have that now.'Humanities, Netherlands

'But it's [i.e. RI education is] also something that even senior people should occasionally have. Have a chance to go and listen to some type of education. Because these things change and the requirements that you are facing are changing, and fields are changing. So maybe, for example, later when we come to this open science and things, maybe, senior people may not know this and it can also be a problem for them so I think they should get the opportunity to get educated.'-Senior researcher, natural sciences, Croatia

'Even though you're a researcher and you have a PhD degree, you follow the, the steps of advancement, it's lifelong learning. And you get $\mathrm{PhD}$ students who are experts in their little field and you're a supervisor, but you may not know all about what they're doing. So, I think it's a matter of acknowledging that we also have[...] competence [to] develop, just as everyone else.'-Senior researcher, medical sciences, Denmark

'it's really, really hard to train the PIs and that is what we are facing now at the ethical review board that a lot of the resistance to these new kinds of procedures is with the more experienced researchers, but, it is really hard to get them trained.'-Secretary of RE committee, natural sciences, Netherlands

'And also for the, for this decision makers or the deans, for the rectors, there should be also written something because they are ruling the, they have a large responsibility. If they fail then they can create larger problem.'Senior researcher, natural sciences, Croatia

Table A.4. Example quotes about the theme 'Education and training approaches, goals and content'.

\begin{tabular}{ll}
\hline Theme/subtheme & Quotes \\
\hline Training goals approach and content & 'I think it's important to cover every aspect of research integrity. At least just to mention it so the, the \\
students, or the, the senior professor know what research integrity is and to bear in their minds what, \\
what all the topics of, of interest there are.'-Junior researcher, natural sciences, Croatia \\
'And when we start with students, we must start on basic things. And we think it is not enough. \\
Because they don't have enough time and place for everything.'-Senior researcher, medical sciences, \\
Croatia \\
'And of course the content of such course depends on the field of research.'-Funder, medical sciences, \\
Belgium \\
'Then align this line [i.e. RI education goals and approach] so at different stages of career and different \\
subjects.'-Junior researcher, social sciences, Germany \\
'And I am not sure if when we talk about fraud being committed is fraud being committed on purpose, \\
which is a criminal issue, or whether it has to do with ignorance. For example, many students com- \\
mit plagiarism without realizing it[...]For me what we need is education'-Senior researcher, medical \\
science, Greece
\end{tabular}


Table A.4. (Continued)

Theme/subtheme Quotes

'We have these workshops on scientific integrity and it's about fraud cases and everyone, at least young $\mathrm{PhD}$ students, and they get scared and they think "That's really bad, I would never do anything like that"'.-RE committee secretary, natural sciences, Netherlands

'The other side of the coin, it's exactly the same talking about misconduct or bad practices because when I plan for what is good practice it means whatever isn't bad.'-Junior researcher, social sciences, Germany

"This is to me saying like: "Okay, this is bad okay". But not really providing the opportunities or not changing anything that drives people towards that behavior'.- Senior researcher, social sciences, Netherlands

Focusing on knowledge of best practices, rules, and regulations

Inspiring and internalizing RI

Addressing daily struggles and gray areas of research
'I think that's very important[...] and expecting these things to be run by host institutions[...]so not putting the full responsibility for this on individual researchers but on the institution, and having the institution run different procedures...training, audits or whatever, to make sure that all its research performing employees are actually aware of current rules or changes in rules.'-Funder, medical sciences, Denmark

'make sure that everyone is aware of what the rules about authorships are.'-Senior researcher, medical sciences, Croatia

'We are higher education researchers and from my everyday research practices there's always the question of citation practices: How to do citations correctly? A very big issue is when you publish a paper and working with quantitative data, quantitative analyses. There are probably guidelines regarding transparency and data, depending on the journal, but they are very different.'-Junior researcher, social sciences, Germany

'often the rules are conducted or taught to students in a such way that they are ambiguous and not detailed enough. So when you actually try to apply them or go deeper and study them, then you, at least in my experience, what you see in studying them is that they were presented to you in mostly the wrong and oversimplistic way.'-Junior researcher, medical sciences, Croatia

'I think it's true that now it's flipped over too much on the forms and bureaucracy and rules. But I think there's also been decades where there was too little emphasis on the ruler.'-Junior researcher, social sciences, Netherlands

'and also it doesn't help to be too prescriptive because I have little bit of fear of the situation that is present at some foreign universities and maybe especially in USA or in some, let's say countries in which law is very strict and in details[...]I think it is not good situation that you have to go to committees before you are allowed to do your research. It would be too stifling for creativity; very, very bad. And our present situation, in my opinion, is not very bad regarding this framework because we have some ethical guidelines and other literature and maybe this kind of education, discussion is something that could be, that could advice more inform people about that topics.'-Senior researcher, humanities, Croatia

'now through research integrity, the whole discussion comes from another side. Its entering the university, not because from the inside we believe in these things but well, you know, our professional[ism] is under threat and the public imagery is turning on its head and I think when it comes to norms and values we do need bigger stories, stories which anchor these values.'-Senior researcher/RI committee member, humanities, Netherlands

'you learn[...]that [rule following] is making intrinsic, originally intrinsic motivation extrinsic, which means that you no longer want this, you don't understand this, you're no longer embodying whatever we're talking about.'-Senior researcher, social sciences, Netherlands

'if there is any cognitive effect on moral behavior, it should have to do with seeing, really seeing that some things are valuable in themselves, or at some end, worthwhile to pursue, not because they're instrumental for another reason. So this requires some sort of, really in depth moral reflection.'Senior researcher/RI committee member, humanities, Netherlands

'But there's a whole range of gray area where as a researcher, especially young researcher, you sometimes don't really know what is right or what is wrong. So I would be very happy to be additionally educated better, what are the rules with outliers, what is the best way, what are the options?'-Junior researcher, social sciences, Netherlands

'This could be a real problem because sometimes when you have project, day-to-day work, practice issues can contradict legal, ethical or whatever issues.'-Junior researcher, social sciences, Germany

'We get many contacts from researchers that are in doubt, "Is this correct to write like this?"'Research integrity officer, natural sciences, Denmark

'Because also sometimes it doesn't only depend, of course, on disciplines, but also on journals you want to publish in and so on and so on, so rules are not fixed, so it's not always easy to give researchers the correct information about the questions they have about it.'-Valorization officer, medical sciences, Belgium

'But if you focus more on specific topics where they are really, you know, in their daily practice they have issues with it, I think it's easier to attract [researchers].'-Senior researcher/Research director, medical sciences, Belgium

'[...] on the one hand there's nothing wrong with having courses that are idealizing the situation [...] That's okay if you have both sides [...] I'm not against my students having these courses, but I do want to tell them that now they need to kind of meet reality. That needs to enter the teaching, or education.'-Senior researcher, social sciences, Netherlands 
Table A.4. (Continued)

\begin{tabular}{|c|c|}
\hline Theme/subtheme & Quotes \\
\hline $\begin{array}{l}\text { Empowering and supporting } \\
\text { researchers }\end{array}$ & $\begin{array}{l}\text { 'something that isn't mentioned in this ethics game, like how do you deal with the fact that you're actu- } \\
\text { ally, as a PhD student, subject to certain power dynamics that you really have very little say in[...]So } \\
\text { it's just students doing it on their own time, really, they're a kind of.. become tennis balls.'-Senior } \\
\text { researcher, humanities, Netherlands } \\
\text { 'It has to do with raising awareness, too. Just knowing what rights you could have if it was an ideal } \\
\text { world. That helps as well.'-Junior researcher, social sciences, Germany } \\
\text { 'We can also train our students, to have, sorry for the gender, for the lack of a better gender, to } \\
\text { have balls, female or male to stand up for their thing. And without that you cannot become a good } \\
\text { researcher.'-Senior researcher, social sciences, Netherlands } \\
\text { 'But maybe just to comment on the discussion about power dynamics: I think it would be good then } \\
\text { just to make PhD students aware really from the very start what the guidelines are and what their } \\
\text { options are, kind of, to empower them from the beginning of their.. before they start publishing, so } \\
\text { maybe some education or training in that regard would help.'-Junior researcher, social sciences, } \\
\text { Netherlands }\end{array}$ \\
\hline Building a culture of integrity & $\begin{array}{l}\text { 'The only thing that should be mandatory is that there's a culture of research integrity and there are } \\
\text { all kinds of instruments that you can think about to promote this culture, and so at [institution } \\
\text { name], that's why we made it [i.e. RI training] mandatory for PhD students and PhD professors, but } \\
\text { in an ideal world you would not have any ethics training but the culture of integrity, that you have } \\
\text { disciplined.'-Senior researcher/RI committee member, humanities, Netherlands } \\
\text { 'I think it's more beneficial to try to create a common culture that people are socialized into and under- } \\
\text { stand what the expected standards are than to try and monitor at every step, which is what I think is } \\
\text { happening now, whether they are applying, whatever rules exist as the should be, in that sense, I do } \\
\text { find that more productive. Make sure people have the same standards, same expectations.'-Senior } \\
\text { researcher, humanities, Netherlands } \\
\text { 'You need an error culture in order to get better. Meaning what, meaning that you are free and open } \\
\text { to admit errors and talk about them because not everyone who makes mistakes is evil.'-Senior } \\
\text { researcher, social sciences, Netherlands } \\
\text { 'I mean still, I mean maybe it [i.e. RI education] will be helpful anyway at least for the young people, } \\
\text { for the, change the culture, the mentality.'-Senior researcher, natural sciences, Croatia } \\
\text { 'I am super curious how you are going to change an existing culture with a training session'.-Senior } \\
\text { researcher, natural sciences, Netherlands }\end{array}$ \\
\hline
\end{tabular}

Table A.5. Example quotes about the theme 'Motivations and incentives for participation in RI education'.

\begin{tabular}{|c|c|}
\hline Theme/subtheme & Quotes \\
\hline Motivations and incentives & - \\
\hline Attractiveness of training programs & $\begin{array}{l}\text { 'The thing with courses is that the people who need it won't do it. It's always the case. } \\
\text { The bad supervisors who are not interesting in supervising, they will not go to a "how } \\
\text { do I supervise PhDs" kind of training. They won't.'-Senior researcher, humanities, } \\
\text { Netherlands } \\
\text { "Research integrity is not what people want to put a lot of time in. And even with doc- } \\
\text { toral PhD students it's very hard to get them to courses of research integrity, they are more } \\
\text { interested in other courses about a subject or other transferable skills courses, but research } \\
\text { integrity is not a favorite. So, we have to force them, let's say, a little bit, to follow these } \\
\text { courses."-Senior researcher/Research director, medical sciences, Belgium } \\
\text { "Here it's where it lies on their priority list, right? It's just not, like you say, they; everyone, } \\
\text { everyone thinks that they are, that they have good integrity..'I don't have three hours of } \\
\text { afternoon to dedicate to this; I have to fly to this conference, } \\
\text { I have to do this, I have to.' So, maybe it's just not priority...”- Research support staff, } \\
\text { natural sciences, Netherlands } \\
\text { 'Senior researcher 1 : Make it easier by dropping the term. Because this is exactly the reli- } \\
\text { gious idea now: Oh there are people who are good, and there are people who are bad, and } \\
\text { we don't want to talk about this. So, learn the right thing, and then if you follow, if you're } \\
\text { a follower then you're a good person, then you will get to heaven[...] } \\
\text { Senior researcher } 2 / \text { RI teacher: You want to facilitate those courses, I think there was a } \\
\text { good comment about maybe not naming them some black and white term, so maybe just } \\
\text { scientific conduct' instead of “integrity course” or "moral thing” or- It's doing research, } \\
\text { that's what it is.'-Social sciences, Netherlands } \\
\text { 'Senior researcher: Yeah, so make it very recent. So, maybe make a promise like in the train- } \\
\text { ing will be the, I don't know, the last five years grey-issues that came up, something like } \\
\text { that. } \\
\text { RE committee secretary: People want it like case studies. } \\
\text { Privacy officer: Which is maybe a little bit like gossip, you know, people are interested in } \\
\text { gossip. So, what came up in that company? Ok. }\end{array}$ \\
\hline
\end{tabular}


Table A.5. (Continued)

Theme/subtheme

Integrating RI education into existing structures
Quotes

RE committee secretary: I recognize that, sort of like the case studies that makes it really alive, and then they can also relate it a little bit easier to their own work.'-Natural sciences, Netherlands

'I've been thinking about a couple of things here, but mainly I think it should just be in the existing things that are in place, like existing forms, the whole fabric of the science that we do. We have a yearly evaluation talk with our supervisor, everybody has that no matter your level, right? Also the dean. If that form for instance would say "What did you do this year to put quality over quantity?" for instance, and you can give any example there, just make sure that you have some quality mark. Maybe you joined a course on integrity...'Senior researcher/RI teacher, social sciences, Netherlands

'And you can also use that competitiveness [in research]. So, the reason that it is competitive is because there is a reward for doing it, like that. You can do a similar thing implementing around the ethics part. Make that also part of; so that you can actually get a reward out of it.'-RE committee secretary, natural sciences, Netherlands

'It will surely create a lot of resistance [if funders require that the PI on a project should go through some sort of research integrity course]'-RI officer, natural sciences, Denmark

'Yeah, but is it important [to require from RPOs that these things are in place before giving out funding]? I'm not sure whether it's important, I would see it as an incentive to take a course in research integrity and so on, but I could see it as an obstacle actually.'-Senior researcher, social sciences, Denmark

'I can tell you in our school, before you become assistant professor or docent one of the prerequisites is that you do a so called teaching practice course that goes on for three days. Because, you know, when you become assistant professor you'll be giving a lectures and this is some sort of preparation for that. Why shouldn't $t$ we introduce a course for the research integrity, AS WELL to that. And make that [a] prerequisite. Because, you're expected to be a supervisor of either, you know, the final, the thesis or $\mathrm{PhD}$ programs When you become full professor, in order to get, to get that title well you have to the management course. Because once you're full professor you have a potential to become a head of the department, or even associate professor. So in that academic promotion, in those steps, well let's introduce obligatory courses that people have to, that people have to do it. There's no negotiation about that. You have to tick that. I've done it and I've got a document saying that I've done it.'-Junior researcher, medical sciences, Croatia

'[Whether training should be made mandatory] depends on the kind of training and the kind of review, so again, if you're dealing with projects where you have to work along through a certain code of conduct, then obviously that has to be reviewed and that has to be mandatory, but, you know, if you want to do a yearly review of referencing, then I don't think that necessarily has to be mandatory, referencing and plagiarism.'-Senior researcher, humanities, Netherlands

Table A.6. Example quotes about the theme 'Organization of RI education and design of trainings'.

\begin{tabular}{|c|c|}
\hline Theme/subtheme & Quotes \\
\hline $\begin{array}{l}\text { Organization and design of } \\
\text { RI training }\end{array}$ & - \\
\hline Continuity of training & $\begin{array}{l}\text { 'I think it's very important to have such a reoccurring event because I noticed that the new PhD stu- } \\
\text { dents, so also the PhD students I am advising, when you are talking about stuff, they were like "I } \\
\text { would never do that". And for me, my fifth year, I was like well that might have sometimes occurred. } \\
\text { So I think some things are also, what you mentioned, we know the rules but sometimes it's a gray } \\
\text { area. And I think if you're starting your PhD, there are some things which I would say that would } \\
\text { never happen to me and then think along the way.'-Junior researcher, social sciences, Netherlands } \\
\text { 'So I wanted to say that I think that one class or one course is not enough.'-Senior researcher, medical } \\
\text { sciences, Croatia } \\
\text { 'So that in a reoccurring course throughout all levels, I think it's important to make sure that you cal- } \\
\text { ibrate your compass each time to make sure you can make decisions. Because there's going to be } \\
\text { new decisions, things you haven't thought about.'-Senior researcher/RI teacher, social sciences, } \\
\text { Netherlands } \\
\text { 'even though you're a researcher and you have a PhD degree, you follow the, the steps of advancement, } \\
\text { it's lifelong learning. And you get PhD students who are experts in their little field and you're a super- } \\
\text { visor, but you may not know all about what they're doing. So, I think it's a matter of acknowledging } \\
\text { that [...]we also have[...]competence [to] develop, just as everyone else.'-Senior researcher, medical } \\
\text { sciences, Denmark }\end{array}$ \\
\hline
\end{tabular}


Table A.6. (Continued)

Theme/subtheme

Format of training
Quotes

'[Formal training] only makes sense if there are very specific guidelines that you want people to know about and those guidelines change. If we are not speaking about that kind of framework then I also don't think there is a need for explicit training.'- Senior researcher, humanities, Netherlands

'not every part of research integrity can be trained in a training module or course but has to be internalized by doing. We already discussed aspects of research integrity that relate to rules and regulations that can be learned...but not every aspect of research integrity can be trained explicitly.'-Senior researcher, social sciences, Germany

'look at these walls here, it's not really inspiring, and a picture of a person you think, well that was really a good scientist, a good academic, you can start talking about her or about him, share the stories. This is what.. many people have said beautiful things, give a nice quotation, that's really meaningful, deep, just I mean, this is a bit of a littered environment, right? It isn't easily inspiring but we have been in the midst, we are the heirs of really inspiring people who founded the university, who had big ideas.. well, a quotation here and there, a good picture, conversations about these people over coffee time, that's really interesting, I mean that's what it's..'-Senior researcher/RI committee member, humanities, Netherlands

'I think there's no way, I mean you have to do things in order to understand what that means. Just to further simulate, give examples is not enough. Because, dealing with real things, nothing is as, as, impressive as dealing with real data. It's your data and you are committed and you want something or not or whatever.'-Senior researcher, social sciences, Netherlands

'I think a number of people now mention specific people or they are part of particular groups like descriptive linguistics or experimental linguistics and within their fields they kind of have the person they go to or they know the form. I mean I'm pretty much working by myself and I'm not part of any particular group so for me to find out what these kind of conventional forms are I would have to kind of hear it through the grapevine or kind of find out who uses what. So it would be good to have kind of a single source as well as the data privacy officer and so on. It would be good to have like a website and just know.. or maybe I didn't do enough research but something like, quite sensible to everyone.'-Junior researcher, humanities, Netherlands

'No, I was just thinking, if it should be a course then it should definitely not be like an entire online course, because then it will just be some passive compliance. Then it should be something in kind of a physical space that could facilitate some reflections on a given topic, relevant.'-RI officer, social sciences, Denmark

'I have [the topic] responsible supervision and mentoring [to rank] which I will put here because it's learning by doing. If you're supervised in a correct and responsible way you will behave automatically as you should, so it promotes research integrity indirectly. It's important. [The topic] education and training and research integrity is second for the same reason because it's better to learn rules by doing and really live them than just learn the rules by rote. On top, not every part of research integrity can be trained in a training module or course but has to be internalized by doing.'-Senior researcher, social sciences, Germany

'I think it's very important to have more like counselling types of people, who, you know approaching them, like they are very accessible and like it's the normal thing to go, you know. People, I hear a lot of stories of students mainly coming back with things that are happening, but they don't know where to go. It says on the website there's a committee for things, but that all sounds very formal. So why would you take that step?'-Senior researcher/RI teacher, social sciences, Netherlands

'that kind of training [i.e. formal course] doesn't make sense if the legalistic framework isn't there. So that was a little bit, like proviso, but... because there are very specific things you want people to know about here, everything is a little bit documented, so you can't easily build that kind of training either, I think. I don't know, that's my feeling and I'm not sure...'-Senior researcher, humanities, Netherlands

'I think that these courses should be taught by people who have some research experience, because they have-, they should have been in these gray areas to say, to have real examples of "Okay, this was a problem. I dealt with this, but I don't know if I did correctly. What do you think?"'- Senior researcher, social sciences, Netherlands

'It's going to sound incredibly cynical, but usually there's just such a gap between what they [i.e. trainers] know and what you need to know, because often, as you we're saying before, it requires such medium specificity, or at least, you know, disciplinary specificity. So I think, yes all supervisees should be trained but you then have to, kind of, come up with a trainer, or a training body, that really is aware of what they're talking about, you know, not sort of post-its on a pyramid in a board or whatever.'-Senior researcher, humanities, Netherlands

'we have divided it [i.e. RI teaching] over the people from part of the committee for scientific integrity[...] and we divide these courses over these people[...]because we feel that the people who.. well, have to solve the problem, so to say, also talk to the people who may cause them, but there's many other ways to think about.'-Senior researcher/RI committee member, humanities, Netherlands

'But that would mean then, each and every teacher or course leader should be aware of that. And some sort of authority should be able to educate them so they actually raise their awareness of that some compliance in their curriculum. That would barely vary from subject to subject.'-Junior researcher, humanities, Croatia 
Table A.6. (Continued)

\begin{tabular}{|c|c|}
\hline Theme/subtheme & Quotes \\
\hline Context of training & $\begin{array}{l}\text { 'The engineering department is completely different than social sciences or humanities. From one super- } \\
\text { visor to another everything can change. So, these are things I think we need to take into account when } \\
\text { talking about research integrity.-Junior researcher, social sciences, Germany } \\
\text { 'just to stress that in case this would be promoted it [i.e. RI training] should be very multidisciplinary } \\
\text { again. That it should be broad.'-Senior researcher, natural sciences, Spain } \\
\text { 'I think, so some of people here are also part of the open science community[...] What we aim to do is } \\
\text { also have these kinds of discussions with people to make sure that everybody is involved[...]I'll give } \\
\text { one short example: we are asked to store our data in an archive in [city] and anthropologists are also } \\
\text { asked to do that, but they have a very different relationship with their participants and it's not like } \\
\text { they can just share these things.'-RI teacher/SR, social sciences, Netherlands } \\
\text { 'Of course, of course in Croatia th[ese] issues are related to the capacities basically. Also not just for } \\
\text { what we want to be there. Because of the capacity you know. If you want to fulfil all of this things, } \\
\text { you need to have some, something in your institute and we have nothing basically. It's not neces- } \\
\text { sary[...]that we should have as institution, but it should be done on, on regional level like other } \\
\text { things, for example, for leading projects whatever you know, offices, PR, whatever. It's not necessary } \\
\text { that every institution, small institution should have this. But then you should organize the systems } \\
\text { somehow different.'-Senior researcher, medical sciences, Croatia }\end{array}$ \\
\hline
\end{tabular}

Table A.7. Example quotes about the theme 'Factors influencing the implementation of RI education and training policies'.

Theme/subtheme Quotes

Implementation factors

Individual factors

Supervision

Research environment
'And maybe you can learn all the rules about scientific integrity, but the individual is also important and that's sometimes very difficult to change. The individual characteristics of the people are also important and play an important role in scientific integrity, I think.'-Senior researcher/research director, medical science, Belgium 'But, I think you can train people and having a code is very good to remind people to be, yeah, true etcetera, etcetera. But, in the end if I am a clever researcher and I want to fraud, play; make fraud, I think I can get away with it to be honest. I think, researchers are clever enough to do it. And, I think whatever you will come up with, they will find a way. So, if someone is really maleficent than I think you will get away with it.'-Privacy officer, natural sciences, Netherlands

'I have a colleague[...] and he is Australian and it seems like he has this quite legalistic approach as well, to resolving things and also behaviors within the university environment.'-Junior researcher, humanities, Netherlands

'We train the PhD students in these courses for half a day or one day during four years when they get trained by that professor or PI for four years, and I am pretty sure that there is quite often a PhD student that comes up here with some issue and the professor: "Ah, don't worry about it."'-Senior researcher, natural sciences, Netherlands '[T]here's very interesting research done by[...] and one of [...]findings was that the stronger the norms were vocally enforced the more unethical the behavior became because people were afraid to report mistake etc. etc. and so the behavior of the manager just above you was key to promoting integrity because you would copy that behavior. But, of course, you have to have the knowledge of, well, what the norms are, so I think we wouldn't have to overstate the importance of these trainings but we have to have them.'-Senior researcher, humanities, Netherlands

'I have responsible supervision and mentoring which I will put here because it's learning by doing. If you're supervised in a correct and responsible way you will behave automatically as you should, so it promotes research integrity indirectly.'-Junior researcher, social sciences, Germany

'Now I would like to come back to the training part because personally I did not have the chance to meet such cases of important fraud. What I have seen in all levels in France and in Greece, is the students not knowing. Misconduct happens, I have seen that, I would say minimum once a year. Very frequently, this is a problem of training we have to talk about it as well, the research environment, the creation of pressure, a good competition between labs (who is going to be the first).'-Senior researcher, medical sciences, Greece

'The way that this is done here is at the lab-level. So it's labs that trains the students, the PhD students, master students, in the way they do things. And this creates a lot of diversion. So so, there are some groups that are very aware of the problem, some others don't care so much, they just look for productivity. And that happens everywhere, I think. So so, the the research integrity in some in some labs is not one thing that they really care about. I'm not saying they don't apply some basic rules, I'm just saying that it's not something they have carefully think about.'-Senior researcher, natural sciences, Spain

'If you don't have the dialogue and the people are feeling like I cannot discuss that I am insecure about this, then you will actually end up with cases that might have gone wrong, simply because the researcher was not sure about the right track and didn't dare to ask. So, it should probably more be about allowing all to learn, rather than the blaming culture.'-Industry representative, natural sciences, Netherlands 
Table A.7. (Continued)

Theme/subtheme Quotes

'Also, creating an internal atmosphere where that, that doesn't help people go in that direction. So, many PhDs may feel the only way for me to get this pressure off from my PI, or this constant like "Where is my Nature paper? Where is my science paper?”... yeah, helping inform or train PIs of how to create an atmosphere that doesn't push people into, into that way.'-Research support staff, natural sciences, Netherlands

'What's happened to the university system in the Netherlands, in general since, sort of, neo-liberal turn, right, we're dealing with a culture that is ultimately focused on a structure of competition at the moment and particularly the discourse of excellence, and these kinds of things are breeding a particular kind of research environment and also an emphasis on, you know, a sort of mythology maybe, of what is valued and what isn't, and I think that if you're really wanting to take the big perspective on that, that is actually really killing a lot of, you know, research integrity in a different kind of way... it's the vanity of the excellent discourse.'-Senior researcher, humanities, Netherlands

Trust versus oversight

'You should have that. The some committee of university or administrate, comes and says "Let me see your document". Although it looks like a bureaucracy the key is in the documents. If you don't have documents, you have nothing.'-Senior researcher, medical sciences, Croatia

'So, you have to train, I think training is the best way to engage; to have responsible researchers, instead of saying: You have to do this, you have to do this, you have to do this as a funder. I think, it also creates more distrust towards, towards researchers instead of the thing you want to achieve [which] is to have responsible researchers.'-Privacy officer, natural sciences, Netherlands

'For me what we need is education and then we have to clear about the later point of implementation. But we must not ignore the educational, cultural point. I think this is very important, otherwise if you not cover the previous step of culture and education, we end up with simple check lists and we should avoid that.'-Senior researcher, medical sciences, Greece

'People are trusted and that's where I see the lack of level of trust here. They're given the training, you might say, and then trusted that they are all doing their job properly without the need to, every time, three, four times a semester, check whether they are applying what they should be.'-Senior researcher, humanities, Netherlands 'But in general requiring courses is part of a general suspicion against people working here, and I want the best for my group, and for the people in my group and I have no interest in harming anybody, neither with my research nor with my colleagues. I may take on decisions but I don't know whether a course would really be the answer to that.'-Senior researcher, humanities, Spain

Reward and incentive

'I think it also has to do, something with structures. If the structures are not good then you can train as long

structures researcher/confidential counselor, humanities, Netherlands

'Yes, definitely. I always think that it's not a lack [of awareness] of guidelines, but the way that the pressure upon researchers and the way that researchers are rewarded or not rewarded by funding, by other things, that causes most of the problems, that's in my personal opinion.'-Senior researcher, medical sciences, Belgium

'That we push our researchers to publish, publish, publish and that they get the salary, let's say, that pays on the number of publications that they publish every year...This is, it is enormously important to do something about that in Europe, especially the young PhDs and Postdocs who are really suffering from short-term contract and short-term contract, and trying to get a scientific job. They are really under pressure to perform and to give their best results. So, when you come in the grey-area they are stretching the grey-area maybe. And they are very; it is very easy for them, not easy for them but it is easy to push them across the border because of this pressure, and I think that is mainly something; we should try to do something in Europe. Because, the system is killing the whole community I guess.'-Funder, natural sciences, Netherlands

'Senior researcher: No, it [i.e. competitiveness] is not a barrier for training.

Funder: It's not a barrier for training.

Senior researcher: It's even, even more

[...]

Funder: You should, because this is a very competitive, so, environment, you need to train and need to get people aware, so that...

Secretary of RE committee: And you can also use that competitiveness. So, the reason that it is competitive is because there is a reward for doing it, like that.'-Natural sciences, Netherlands 